\title{
Influence of antioxidants in epoxy-anhydride resin used for HV applications
}

\author{
Chau Hon Ho, Emmanuel Logakis, Andrej Krivda \\ ABB Switzerland Ltd., Corporate Research, Segelhofstr. 1K, 5405 Baden-Daettwil, Switzerland
}

\begin{abstract}
In this work, various antioxidants with different chemical structures and functionalities were investigated in respect to their influence in the processibility and properties of a standard epoxyanhydride resin formulation used in HV applications.

Such antioxidants are mainly used in thermoplastic materials as they are known for their long- and shortterm stabilizing effect against thermal and thermooxidative degradation. They are also used as UV stabilizers against decomposition and discoloration of the polymer and as metal deactivators by catching and eliminating metal ions. Recently, the use of such antioxidants as voltage stabilizers in cross-linked polyethylene has been reported showing a significant increase in breakdown voltage.

Our investigations show that the processing requires additional mixing steps in order to completely dissolve antioxidants in the epoxy formulation. While little amounts of antioxidants or voltage stabilizers have a large influence in thermoplastics regarding thermal stability and electrical properties, the studied antioxidants in a concentration range of $1-5$ weight- $\%$ show surprisingly only little impact in breakdown voltage as well as in dielectric properties and also in degradation behavior as detected by thermo-gravimetric analyses. Only the glass transition temperature of the cured epoxy resin is decreased in a few cases where the antioxidants have a plasticizing effect.
\end{abstract}

\section{Introduction}

Epoxy-anhydride thermosets are known for their excellent combination of thermo-mechanical properties combined with superior electrical insulation properties and are widely used - especially in high voltage (HV) applications [1][2]. In addition, epoxy-anhydride materials demonstrate superior chemical resistance and high thermal stability.

A large variety of technical approaches have been tried to further enhance the properties of epoxy-anhydride systems. In particular epoxy nanocomposites based on inorganic fillers such as silica or alumina have been studied extensively in the recent years [3][4]. While maintaining good processibility with low viscosity of the resin containing nanosized fillers, substantial improvement of electrical properties - e.g. mitigation of space charge build-up [5] and increased AC breakdown strength [6] - has been reported. However, a major drawback of the use of inorganic solid nanofillers is the large influence of dispersion quality in the liquid epoxy resin and the final cured composite [7]. This may lead to inconsistent product quality in case of final implementation of nanocomposites in power products. An alternative route would be the use of organic low molecular weight additives which may boost the properties of the epoxy materials. Such organic substances can be fully dissolved and therefore perfectly dispersed in the epoxy resin. Antioxidants are widely used in thermoplastic materials as they are known for their long- and short-term stabilizing effect against thermal and thermo-oxidative degradation. They are also used as UV stabilizer and as metal deactivator. In particular the so-called primary antioxidants, such as hindered phenols or aromatic amines, are essential for long-term stability as they work as free radical scavengers in the polymer degradation mechanism.

A few studies have shown the influence of such antioxidants in thermoplastics such as polyethylene in respect to electrical properties [8][9]. The increased dielectric strength may be related to the change of polymer morphology, crystallinity and spherulite size [10]. In addition, other studies claim that antioxidants such as benzophenone or carbazole and their derivatives may function as so-called voltage stabilizers improving the breakdown performance. According to the latter, antioxidants acting as electron scavengers are able to catch fast high energy single electrons in the electric field [11]-[13].

The influence of antioxidants on electrical performance in epoxy-anhydride systems or epoxy thermosets in general has not been studied yet. In this work, various antioxidants - typically used in thermoplastics - with different chemical structures and functionalities were studied in respect to processibility and their influence in the properties of a standard epoxy-anhydride resin formulation used in HV applications.

\section{Experimental procedure}

\subsection{Materials}

As thermoset matrix, a standard epoxy-anhydride resin formulation used in $\mathrm{HV}$ applications was used. The studied antioxidants, their name and chemical structure are shown in Table 1.

Table 1 - List of studied antioxidants

\begin{tabular}{|l|l|}
\hline Chemical structure & Trade name (CAS) \\
\hline
\end{tabular}




\begin{tabular}{|l|l|}
\hline \\
\hline
\end{tabular}

\subsection{Cast sample preparation}

Bisphenol $\mathrm{A}$ and the amine catalyst were dissolved in the anhydride at $100{ }^{\circ} \mathrm{C}$ in an oven for $4 \mathrm{~h}$ using a standard propeller mixer. The mixture was cooled down to $60{ }^{\circ} \mathrm{C}$ and the antioxidant ( $\mathrm{x}$ wt- $\%$ in addition to the final mixture) was added and mixed. Then the preheated epoxy resin was added. The final mixture was degassed and stirred at $10 \mathrm{mbar}$ for $20 \mathrm{~min}$ at $60^{\circ} \mathrm{C}$. After casting into appropriate steel molds, the mixture was let for another $20 \mathrm{~min}$ at $5 \mathrm{mbar}$ at $60{ }^{\circ} \mathrm{C}$ and eventually cured at $120{ }^{\circ} \mathrm{C}$ for $10 \mathrm{~h}$ and $140{ }^{\circ} \mathrm{C}$ for another $10 \mathrm{~h}$.

\subsection{Characterization techniques}

Dielectric spectroscopy measurements were carried out using a Novocontrol Alpha analyzer in the frequency range from $1 \mathrm{~Hz}$ to $1 \mathrm{MHz}$. Temperature control was achieved by a Novocontrol Quatro cryosystem (range from 40 to $180{ }^{\circ} \mathrm{C}$ ). Specimens of $38 \mathrm{~mm} \times 38 \mathrm{~mm} \times 1$ $\mathrm{mm}$ were cut from cast plates and coated with round thin electrodes (gold over chrome), $30 \mathrm{~mm}$ in diameter, to ensure good electrical contacts.

The glass transition temperature $\left(\mathrm{T}_{\mathrm{g}}\right)$ was measured using a Perkin Elmer DSC-7 calorimeter. Cured samples of ca. $50 \mathrm{mg}$ were loaded in vented aluminum pans. Heating ramps were performed at a rate of $10^{\circ} \mathrm{C} / \mathrm{min}$. The dielectric strength of the cured resins was tested on cast rod-rod samples using step-up electrical breakdown (BD) tests at $50 \mathrm{~Hz}$ (steps of about $1 \mathrm{kV}$ peak per mm per minute). The tests were performed in $\mathrm{SF}_{6}$ at 4 bars. The configuration of the rod-rod epoxy samples is shown in Fig. 1. The surface of the electrodes was sandblasted according to the Rugotest No. 3 class A with an average surface roughness of about $6 \mu \mathrm{m}$. All tested samples were partial discharge free as tested prior to the BD tests according to IEC 60270 standard.

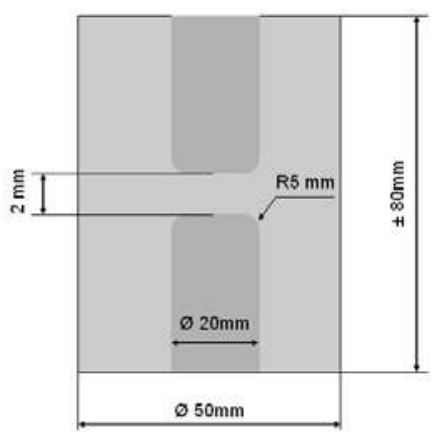

Fig. 1 - Scheme of rod-rod sample used in dielectric BD tests

\section{Results and discussions}

\subsection{Processibility}

The additives Ionol 46 and Irganox 330 are not soluble in either anhydride or final epoxy-anhydride mixture. Interestingly, addition of Ionol LC started polymerizing the anhydride. Therefore, these three substances were excluded from further investigations. All other five antioxidants could be fully dissolved in the liquid resin, and transparent cured samples were obtained.

\subsection{Dielectric constant}

Fig. 2 shows the temperature dependence of the relative permittivity $\left(\varepsilon_{\mathrm{r}}\right)$ for the different formulations with 3 wt- $\%$ antioxidant and the pure resin as reference. In general, no large effect on the permittivity value is observed with addition of antioxidants: $\varepsilon_{\mathrm{r}}=3.5-4.0$ at low and $\varepsilon_{\mathrm{r}}=5.0-6.0$ at high temperature. The abrupt rise in permittivity is related to the glass transition of the epoxy-anhydride matrix.

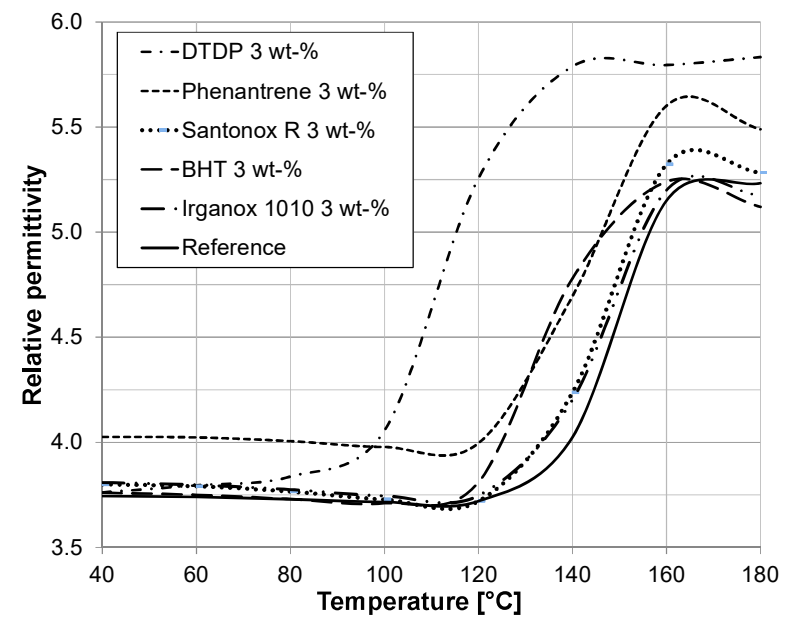

Fig. 2 - Relative permittivity at $50 \mathrm{~Hz}$ of various formulations with antioxidants (all $3 \mathrm{wt}-\%$ ) as well as unfilled reference 
Overall, a slight increase in dielectric constant can be observed which can be attributed to the increased polarity due to the functional groups in the antioxidants. The effect is largest in case of DTDP which contains highly polar ester functionalities. Fig. 3 and Fig. 4 show the relative permittivity for formulations with DTDP and Santonox R, respectively, with varying antioxidant concentration. Besides the increase in polarity it is obvious that DTDP with the flexible alkyl moieties acts also as plasticizer in the epoxy network: the permittivity rises at lower temperature. This can be concluded also from the DSC analyses where a reduction in $\mathrm{T}_{\mathrm{g}}$ is detected, from $128{ }^{\circ} \mathrm{C}$ for the unfilled reference to 107 ${ }^{\circ} \mathrm{C}$ for the material with $3 \mathrm{wt}-\%$ DTDP. In contrast, Santonox $\mathrm{R}$ has only little influence on the dielectric constant.

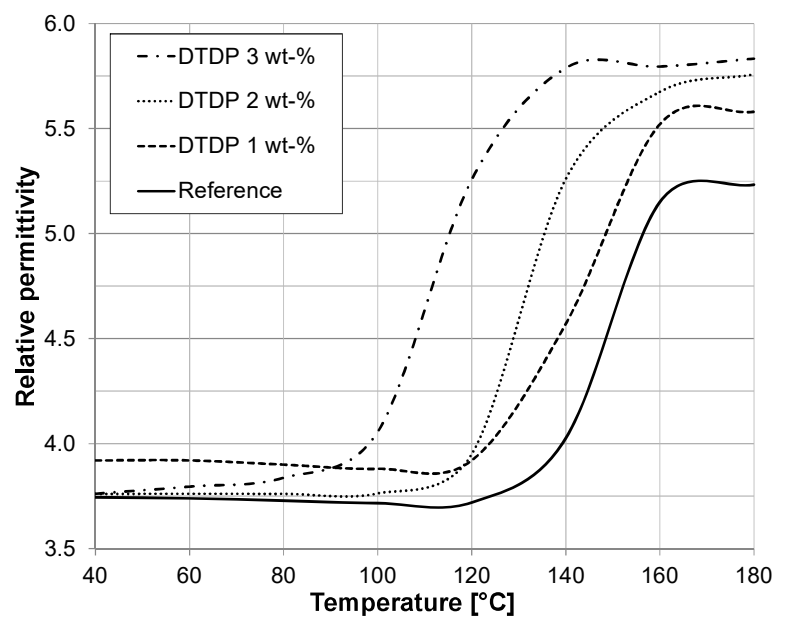

Fig. 3 - Relative permittivity at $50 \mathrm{~Hz}$ of various formulations with DTDP (1-3 wt-\%) as well as unfilled reference

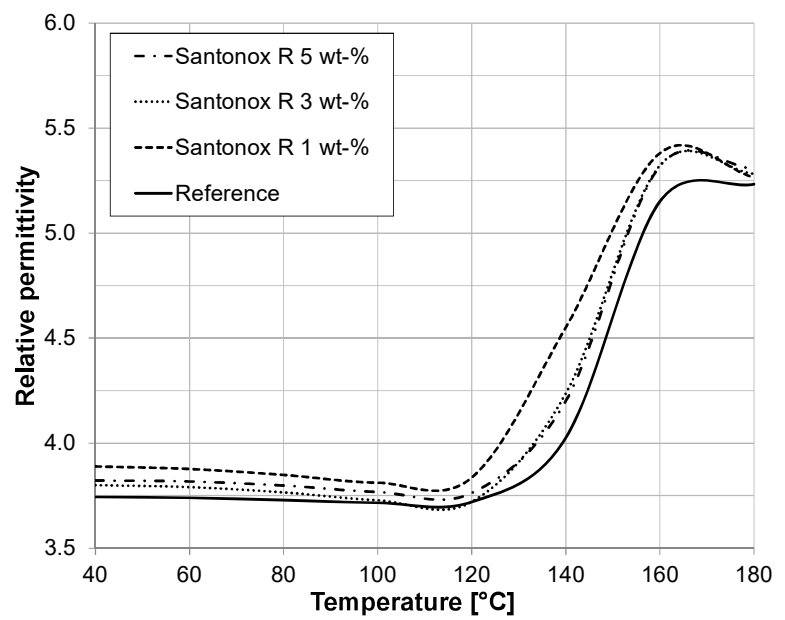

Fig. 4 - Relative permittivity at $50 \mathrm{~Hz}$ of various formulations with Santonox R (1-5 wt-\%) as well as unfilled reference

\subsection{Dielectric loss}

The observations made in the analyses on relative permittivity are similar to the dielectric loss data which are shown Fig. 5. Again, the highest influence is given by the addition of DTDP exhibiting the abrupt rise in dielectric loss at lower temperature. Increasing DTDP concentration leads to a stronger effect (not shown here). As indicated in Fig. 4 and Fig. 5 and the data on $\varepsilon_{\mathrm{r}}$ and $\tan \delta$, BHT may also have a minor plasticizing effect resulting from its rather low molecular weight and small size.

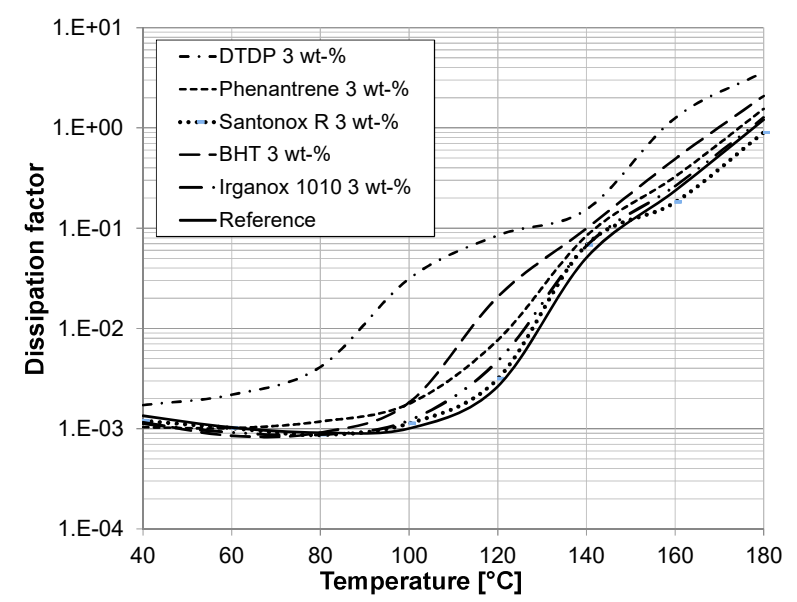

Fig. 5 - Dissipation factor at $50 \mathrm{~Hz}$ of various formulations with antioxidants (all $3 \mathrm{wt}-\%$ ) as well as unfilled reference

\subsection{Dielectric breakdown strength}

The results from the dielectric breakdown tests Weibull statistics according to the White method - are shown in Fig. 6 and Fig. 7. Values for $\alpha$ and $\beta$ are listed in Table 2. From the data in Fig. 6 it can be concluded that the addition of antioxidants ( $3 \mathrm{wt}-\%$ ) has basically no influence on the breakdown strength of the epoxyanhydride resin formulation. However, only the use of DTDP (3 wt-\%) results again in a modification of the electrical performance: a reduction of the breakdown strength of ca. $18 \%$.

Interestingly, samples containing phenantrene show a significantly lower scattering compared to the reference. Phenantrene may act as stabilizer of the epoxy resin during processing and curing prohibiting local hot spots and resulting into better and consistent sample quality. Fig. 7 shows breakdown data in Weibull plots for epoxy formulations containing DTDP and Santonox R, respectively, at various concentrations underlining the negative impact of DTDP on the resin regarding breakdown while Santonox R shows no influence.

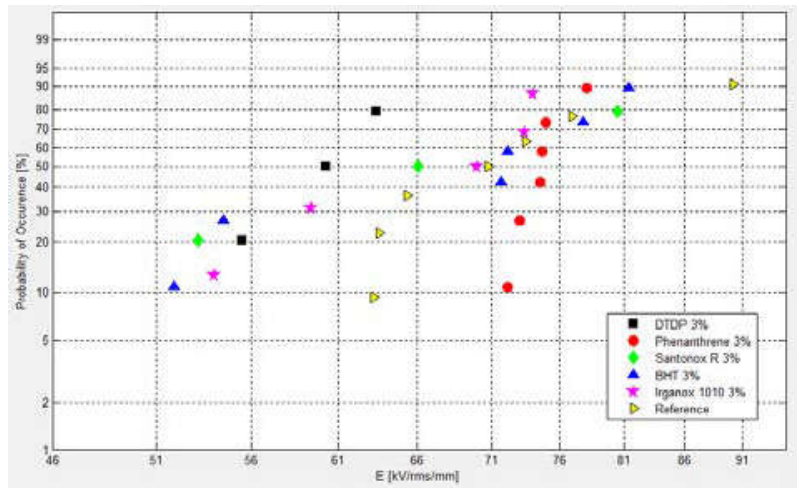

Fig. 6 - Dielectric strength at $50 \mathrm{~Hz}$ of various formulations with antioxidants (all $3 \mathrm{wt}-\%$ ) as well as unfilled reference 


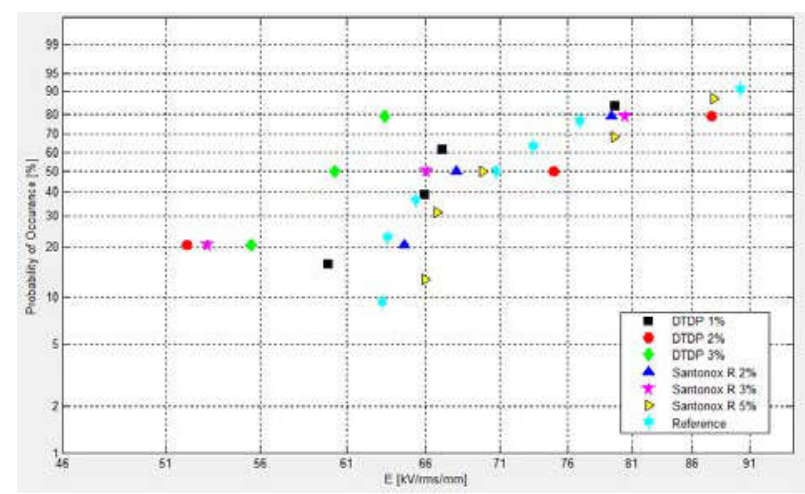

Fig. 7 - Dielectric strength at $50 \mathrm{~Hz}$ of various formulations with DTDP and Santonox R as well as unfilled reference

Table 2 - Breakdown data of various formulations with antioxidants as well as the reference system

\begin{tabular}{|l|c|c|}
\hline Resin formulation & $\alpha$ & $\beta$ \\
\hline DTDP 1 wt-\% & 71.9 & 8.7 \\
\hline DTDP 2 wt-\% & 81.0 & 3.9 \\
\hline DTDP 3 wt-\% & 61.9 & 15.6 \\
\hline Santonox R 2 wt-\% & 74.6 & 9.3 \\
\hline Santonox R 3 wt-\% & 73.5 & 5.0 \\
\hline Santonox R 5 wt-\% & 78.0 & 8.3 \\
\hline Phenantrene 3 wt-\% & 75.5 & 39.8 \\
\hline BHT 3 wt-\% & 73.9 & 5.7 \\
\hline Irganox 1010 3 wt-\% & 70.6 & 7.6 \\
\hline Reference & 75.6 & 8.3 \\
\hline
\end{tabular}

\section{Summary}

Several antioxidants were investigated with respect to the influence of their addition on the electrical properties of standard epoxy thermosets used in HV insulation. Most of the antioxidants show high solubility in the liquid epoxy formulation allowing perfect distribution in the final matrix.

However, the studied antioxidants did not show any improvement in dielectric strength of the epoxyanhydride system as described for thermoplastics in the past. Most likely, the observed properties are related to the change in the morphology and crystallinity of the thermoplastics rather than to any electron scavenger effect - especially considering typical antioxidant concentrations of $<1 \mathrm{wt}-\%$ for thermoplastics.

In general, the introduction of antioxidants led to a slight overall increase of dielectric constant and loss due to the polarity of the additives. Largest effects were obtained with DTDP containing polar ester bonds. In addition, DTDP with its long flexible alkyl chains acts as a plasticizer for the epoxy-anhydride matrix and reduces the glass transition temperature and therefore, shifts the abrupt increase of $\varepsilon_{\mathrm{r}}$ and $\tan \delta$ to lower temperatures.

\section{References}

[1] D. A. Bolon, Epoxy chemistry for electrical insulation, Electrical Insulation Magazine IEEE, 1995, 11, 10-18.

[2] L. Valette, J. Li Yong, D. Ji, K. Karunakaran, Novel high performances epoxy/anhydride formulations and their application in electrical insulation, Electrical Insulation Conference (EIC), 2011, 459-463.

[3] Green, C.; A. Vaughan. Nanodielectrics - How Much Do We Really Understand, Electrical Insulation Magazine IEEE, 2008, 24, 6-16.

[4] T. Tanaka, G. C. Montanari, R. Mulhaupt, Polymer nanocomposites as dielectrics and electrical insulation-perspectives for processing technologies, material characterization and future applications, IEEE Transactions on Dielectrics and Electrical Insulation, 2004, 11, 763-784.

[5] M. G. Veena, N. M. Renukappa, J. M. Raj, C. Ranganataiah, K. N. Sivakumar, Characterization of nanosilica-filled epoxy composites for electrical and insulation applications, J. Appl. Polym. Sci. 2011, 121, 2752-2760.

[6] G. Iyer, R. S. Gorur, R. Richert, A. Krivda, L. E. Schmidt, Dielectric properties of epoxy based nanocomposites for high voltage insulation, IEEE Transactions on Dielectrics and Electrical Insulation, 2011, 18, 659-666.

[7] Z. Su, H. Hillborg, E. Martensson, G. Paulsson, Evaluation of epoxy nanocomposites for electrical insulation systems, Electrical Insulation Conference (EIC), 2011, 489-492.

[8] L. Boudou, N. Zouzou, J. Vega, Conductivity of polyethylene - role of antioxidant and crosslinking by-products, IEEE 7th International Conference on Solid Dielectric, 2001, 245-247.

[9] M. Goshowaki, K. Noguchi, U. Kawabe, Y. Sekii, Influence of antioxidants on electrical conduction in $L D P E$ and XLPE, Journal of Electrostatics, 2007, 551-554.

[10] S. N. Kolesov, The Influence Of Morphology On The Electric Strength Of Polymer Insulation IEEE Transactions on Electrical Insulation, 1980, 15, 382-388.

[11] T. Hjertberg, Voltage stabilizers for XLPE cable insulation, 8th International Conference on Insulated Power Cables, 2011, E728.

[12] T. Person, B. Neese, Voltage stabilizing additive assessment in polyethylene insulation, 8th International Conference on Insulated Power Cables, 2011, C54.

[13] W. Bancouf, X. Colin, C. Monchy-Leroy, G. Marque, Study of the stabilization of polyethylene and ethylene-propylene copolymers by a phenolic antioxidant, 8th International Conference on Insulated Power Cables, 2011, E513. 